\title{
Preoperative assessment of deep myometrial and cervical invasion in endometrial carcinoma: Comparison of magnetic resonance imaging and histopathologic evaluation
}

\author{
C. VASCONCELOS ${ }^{1}$, A. FÉLIX ${ }^{2} \&$ T. M. CUNHA ${ }^{3}$ \\ Departments of ${ }^{1}$ Radiology, Hospital Egas Moniz, ${ }^{2}$ Pathology and ${ }^{3}$ Radiology, Instituto Português de Oncologia de Francisco \\ Gentil, Lisbon, Portugal
}

\begin{abstract}
Summary
This study aimed to evaluate the accuracy of magnetic resonance imaging (MRI) in the detection of deep myometrial invasion and cervical extension by endometrial carcinoma. This prospective study included 101 patients with histologically documented endometrial carcinoma, between July 1998 and April 2004. The findings of preoperative pelvic MRI were compared with histological diagnosis. From 101 cases studied by pelvic MRI, 43 were classified as deep myometrial invasion ( $\geq 50 \%$ of myometrium), where the pathological evaluation confirmed as having deep myometrial invasion. Cervical extension in the MRI study was found in 19 cases. Pathologic study found cervical extension and/or invasion in 31 cases including all cases identified by MRI. The accuracy, sensitivity and specificity of MRI were $95 \%, 89 \%, 100 \%$, detecting deep myometrial invasion and $88 \%, 61 \%, 100 \%$, detecting cervical invasion, respectively. The high accuracy achieved makes MRI an adequate method for determine the depth of myometrial and cervical invasion in endometrial carcinoma.
\end{abstract}

\section{Introduction}

In Portugal, endometrial carcinoma is the second most common cancer of female genital tract (Cunha et al. 2001). Presenting as abnormal vaginal bleeding, the disease occurs most frequently in white women, with peak incidence between ages 55 and 65. Known risk factors are unopposed oestrogen intake, tamoxifen, nulliparity, obesity, diabetes and the Stein-Leventhal syndrome (Belloni et al. 1990).

In 1988, the International Federation of Gynecology and Obstetrics (FIGO) adopted a surgico-pathological system for the staging of endometrial carcinoma (Anonymous 1989). The rationale behind the change from a clinical to a surgico-pathological staging system was based on the inaccuracy of clinical staging and subsequent limitations of treatment planning (Frei et al. 2000).

Treatment and prognosis depend on several factors, including stage, tumour grade, depth of myometrial penetration, cervical penetration and nodal metastasis (Manfredi et al. 2004; Kinkel et al. 1999; Hricak et al. 1991; Sironi et al. 1992a).

Symptomatic abnormal bleeding occurs in about $80 \%$ of patients, and most of the cases are diagnosed in an early-stage (Sironi et al. 1992a), and cured (Lien et al. 1991).

Because extrauterine disease and lymph node metastases strongly correlates with the depth of myometrial invasion and histological tumour grade (Belloni et al. 1990), preoperative knowledge of myometrial invasion directly influences the choice of treatment (Kinkel et al. 1999).
Patients with grade 3 carcinomas have a high prevalence of lymph node metastases and should be proposed to undergo surgical lymph node assessment (Frei and Kinkel 2001). In most institutions, patients with $>50 \%$ of myometrial invasion are also considered for further surgical staging, including pelvic and para-aortic lymphadenectomy. Therefore, treatment should include intraoperative staging by a pathologist and a gynaecologist with special training in oncological surgery (Frei and Kinkel 2001). Recent data from the ASTEC study, presented at the 37th Annual Meeting of the Society of Gynecologic Oncologists of North America in March 2006, has shown preliminary data that surgical staging by way of pelvic and para-aortic lymphadenectomy does not improve survival (Kitchener 2006). The knowledge of cervical invasion may alter the type of hysterectomy that is performed and the detection of parametrial invasion should raise the possibility of radiation therapy as the first treatment (Kinkel et al. 1999).

Pelvic MRI is an examination accepted in the characterisation of pelvic tumours due to its multiplanar capacity and its excellent resolution of soft tissue contrast (Cunha et al. 2001; Sironi et al. 1992a; Sironi et al. 1992b). High accuracy in the assessment of the depth of myometrial infiltration (superficial/deep) as well as the extent of cervical invasion and identification of enlarged pelvic and para-aortic lymph nodes has been described in the literature (Cunha et al. 2001; Manfredi et al. 2004).

In this prospective study, we evaluate the accuracy of MRI in endometrial carcinoma (invasion of the deep myometrium and of the uterine cervix), compared with surgicopathological findings. 


\section{Material and methods}

Between July 1998 and April 2004, a total of 108 consecutive patients with endometrial carcinoma treated in the Department of Gynaecology of the Portuguese Institute of Oncology, were considered for inclusion in this prospective study. In this series, 40 cases were previously reported in the literature (Cunha et al. 2001).

Inclusion criteria were the presence of endometrial carcinoma histologically documented by biopsy and confirmed on the surgical specimen and a preoperative pelvic MRI.

One patient was excluded from the study because she subsequently underwent surgery at a different institution. Six patients were also excluded because they did not undergo surgery - four were referred to radiation therapy and the other two were treated with hormonal therapy.

Therefore, our study included 101 patients with an age range between 48 and 89 years (mean $=68.5$ years). A total of 11 were premenopausal $(11 \%)$ and 90 were postmenopausal (89\%); regarding exogenous hormonal therapy, three were on contraceptives, eight were receiving hormonal replacement therapy and three were taking tamoxifen for breast cancer. The average time between MRI and hysterectomy was 45 days (range $0-90$ days).

All patients were submitted to abdominal hysterectomy and bilateral salpingo-oophorectomy, with pelvic lymphadenectomy in 36, and selective para-aortic lymphadenectomy in 15.

The histological diagnosis and the tumour grade are shown in Table I. At histological examination, 86 were endometrioid adenocarcinomas, five were papillary serous adenocarcinomas, three were clear cell carcinomas, three were mucinous adenocarcinomas, one was a mixed malignant Müllerian tumour, one was a glassy cell carcinoma and two were undifferentiated carcinomas. In regard to tumour grade, 39 patients had grade 1 tumours, 34 had grade 2 tumours and 28 had grade 3 tumours. The FIGO stages were as follows: 7 stage IA (7\%), 40 stage IB (39\%), 20 stage IC $(20 \%), 19$ stage II $(18 \%), 11$ stage III $(10 \%)$ and 4 stage IV (4\%).

MRI studies were performed with a 1.0-Tesla superconducting magnet (model Gyroscan NT; Philips Medical Systems, Einthoven, Netherlands), using a body coil. In each examination, axial T1-weighted $(\mathrm{TR} / \mathrm{TE}=550 /$ $14 \mathrm{~ms})$ and axial and sagittal T2-weighted $(\mathrm{TR} / \mathrm{TE}=4090 /$ $150 \mathrm{~ms}$ ) images were acquired. The parameters were two acquisitions, a matrix of $256 \times 256$, with $5 \mathrm{~mm}$ slice thickness and $1 \mathrm{~mm}$ interslice gap.

Table I. Endometrioid carcinoma: histological type and grade

\begin{tabular}{lrrrr}
\hline & \multicolumn{3}{c}{ Tumour grade } & \\
\cline { 2 - 4 } & 1 & 2 & 3 & Total \\
\cline { 2 - 4 } Histopathological type & 36 & 34 & 16 & 86 \\
Endometrioid adenocarcinoma & 3 & & & 3 \\
Mucinous adenocarcinoma & & & 5 & 5 \\
Papillary serous adenocarcinoma & & 3 & 3 \\
Clear cell carcinoma & & & 2 & 2 \\
Undifferentiated carcinoma & & & 1 & 1 \\
Mixed malignant Müllerian tumour & & & 1 & 1 \\
Glassy cell carcinoma & & & & \\
\hline
\end{tabular}

Dynamic study after rapid manual intravenous administration of $0.1 \mathrm{mmol} / \mathrm{kg}$ gadopentetate dimeglumine (Magnevist; Schering, Berlin, Germany) was performed using a fast field-echo pulse sequence, with multisection in the sagittal plane of the uterine corpus. The parameters were $16 / 5.3 \mathrm{~ms}(\mathrm{TR} / \mathrm{TE})$, a flip angle of $25^{\circ}$, a $256 \times 256$ matrix and an acquisition of images at equilibrium phase, $90-120 \mathrm{~s}$ after the injection of the contrast material.

MR images were independently analysed prospectively by two radiologists (C.V., T.M.C.) prior to surgery. Interpretation discrepancies were resolved by consensus. The images were interpreted for the following parameters: depth of myometrial invasion, cervical invasion and lymph node enlargement.

Myometrial infiltration was classified in superficial ( $<50 \%$ of the thickness of the myometrium) if there was disruption or discontinuity of the junctional zone in the $\mathrm{T} 2$-weighted images or when the internal surface of the myometrium was irregular in the dynamic study (Figure 1), and in deep invasion $(\geq 50 \%$ of the thickness of myometrium) when the tumour was observed reaching the external half of the myometrium (Figures 2 and 3). Regular thinning of the myometrium was classified as superficial invasion (Figure 4).

Cervical extension was determined when widening of the internal os and cervical canal and/or as abnormal signal intensity in the cervical canal; cervical epithelium disappeared partly or totally and/or disruption of the normal low signal intensity of junctional zone in the cervix on sagittal $\mathrm{T} 2$-weighted images was visualised. In the dynamic study, the cervical infiltration was considered when interruption of the contrast enhancement of the epithelium (Figure 5) or the cervical stroma (Figure 6) was observed.

MRI findings were compared with surgico-pathological results, and the diagnostic accuracy, sensitivity, specificity, positive and negative predictive values were calculated for myometrial infiltration and cervical invasion by endometrial carcinoma [sensitivity $=\mathrm{TP} /(\mathrm{TP}+\mathrm{FN})$, specificity $=\mathrm{TN} /(\mathrm{TN}+\mathrm{FP})$ and accuracy $=(\mathrm{TP}+\mathrm{TN}) /$ $(\mathrm{TP}+\mathrm{TN}+\mathrm{FP}+\mathrm{FN})]$, where $\mathrm{TP}$ is the true positive, $\mathrm{TN}$ is the true negative, $\mathrm{FN}$ is the false negative and FP is the false positive (Glantz 1981; Landis and Koch 1997).

\section{Results}

In our 101 cases studied, according to surgico-pathological data, $\geq 50 \%$ of myometrial infiltration was found in 48 cases, $11 \mathrm{had}$ cervical extension to the epithelium and 20 had stromal invasion. MRI correctly assessed 43 (90\%) patients with deep myometrial invasion and underestimated five cases $(10 \%)$. Among the five understaged cases, one had an 80-day interval between MRI and the surgery and another was a grade 3 neoplasm. In two others cases, the evaluation was difficult, by the presence of an intramural leiomyoma and by the polypoid growth of the tumour. In the last case, invasion of the external half of the myometrium consisted of penetration by small isolated glandular foci.

Overall, MRI sensitivity, specificity, diagnostic accuracy and positive and negative predictive values for deep myometrial invasion was $89 \%, 100 \%, 95 \%$ and $100 \%, 91 \%$, respectively.

Regarding cervical extension, MRI found invasion in $19(61 \%)$ cases. Histopathological study detected cervical 

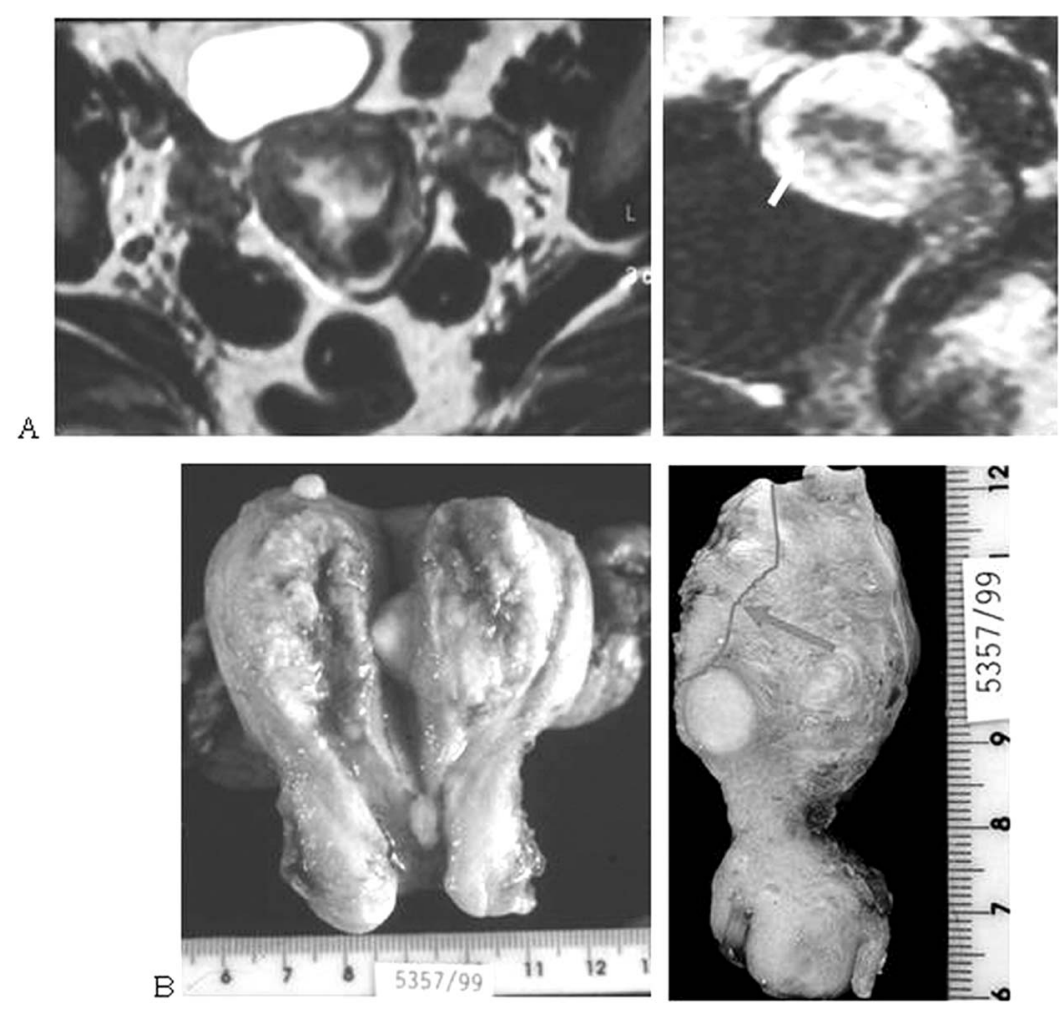

Figure 1. Images of a 55-year-old woman with superficial myometrial invasion. (A) Axial T2-weighted image (left) shows an expanded central zone of high signal intensity with irregularity of the junctional zone, indicating invasion of $<50 \%$ of the thickness of the myometrium. On the sagittal dynamic MR image, after contrast enhancement (right) the demonstration of the internal surface myometrial invasion is better observed than on the T2-weighted image by the irregular interface between the tumour and the myometrium (arrow). (B) Gross specimen of hysterectomy with bilateral salpingo-oophorectomy and sagittal section of the uterus, with a polypoid mass invading the superficial myometrium (arrow). An intramural leiomioma in the uterine corpus is also documented.
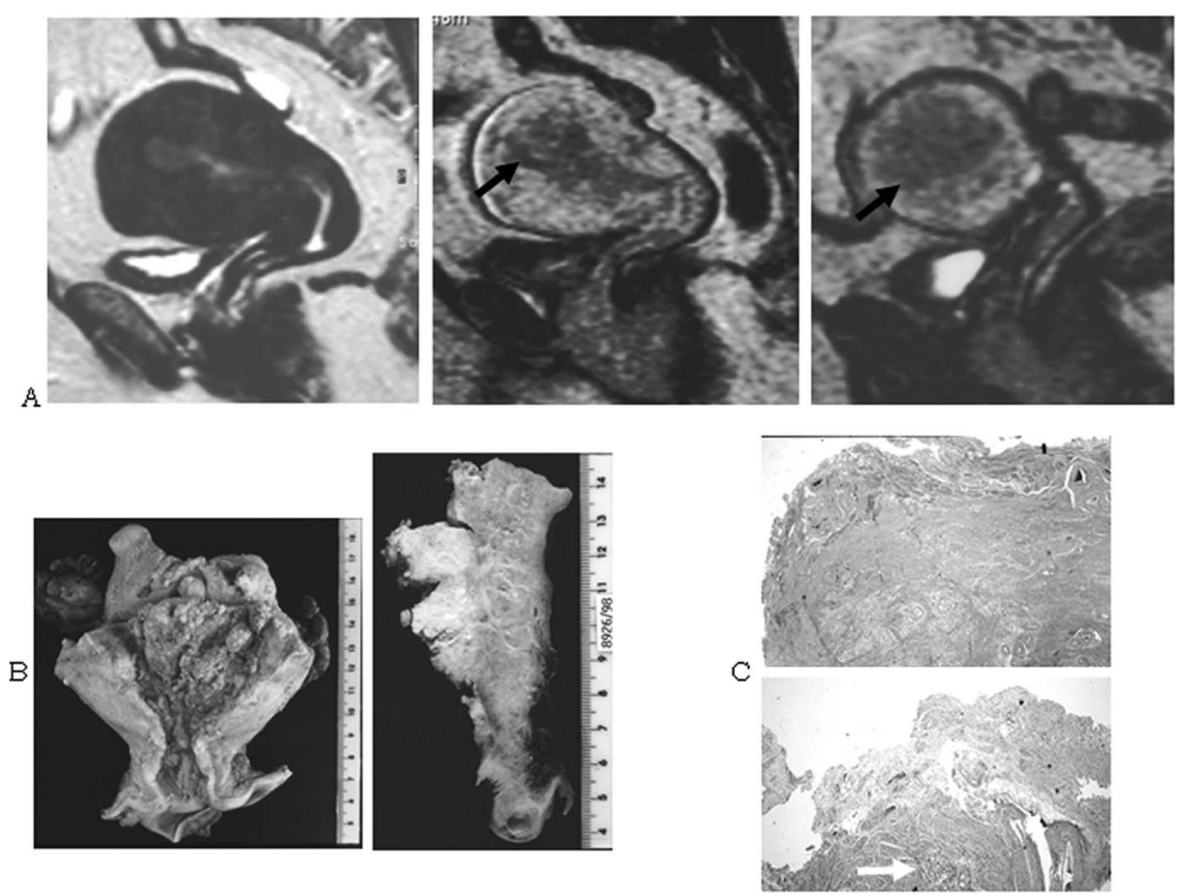

Figure 2. A 62-year-old woman with deep myometrial invasion. (A) Sagittal T2-weighted image (left) and sagittal dynamic gadolinium enhanced images (centre and right). The demonstration of the tumour extending into the outer half of the myometrium (deep myometrial invasion) is better identified in T1-weighted images after contrast (arrows) due to the presence of adenomyosis documented in the T2-weighted image. (B) Gross specimen of hysterectomy with bilateral salpingo-oophorectomy and sagittal section of the uterus with a polypoid tumour occupying the endometrial cavity and invading the deep myometrium. (C) Histological section of the uterine wall with an adenocarcinoma invading the external half of the myometrium (arrow). 

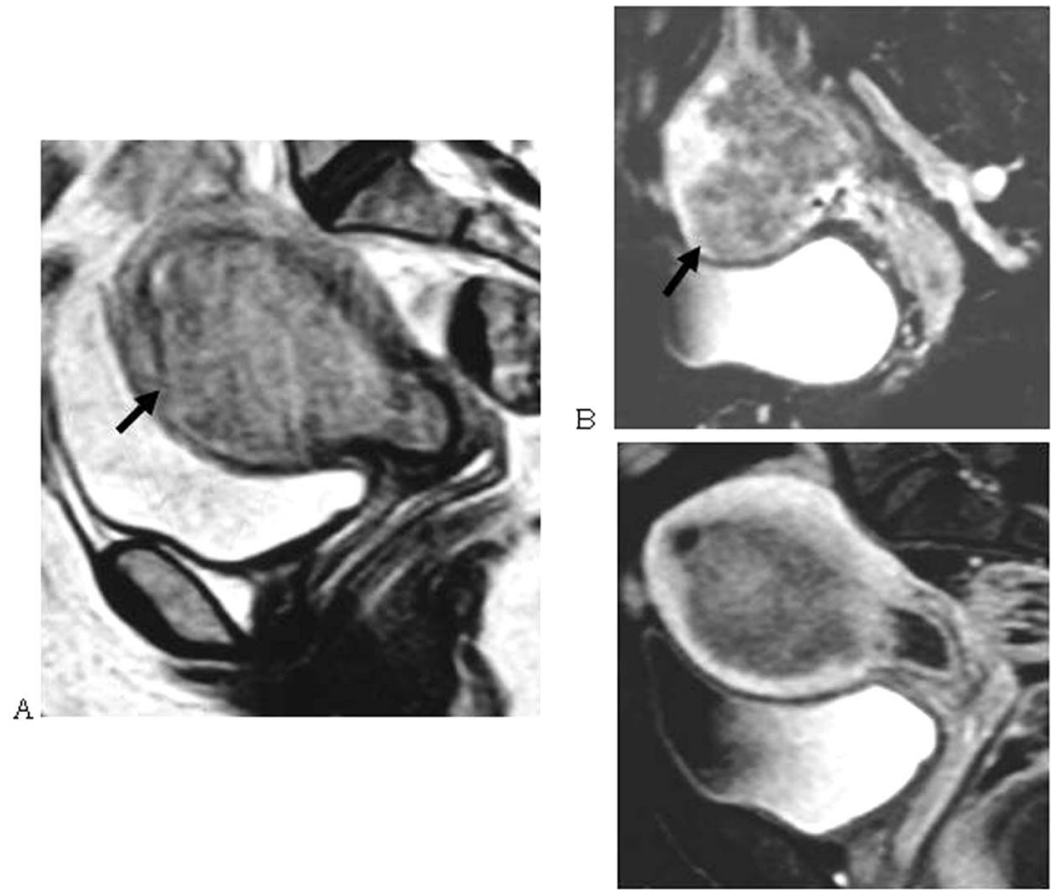

Figure 3. Histological stage I endometrial carcinoma with invasion of the outer half of the myometrium. (A) Sagittal T2-weighted image and (B) sagittal dynamic MR images after contrast. The uterus is mostly occupied by the tumour with irregular thinning of the myometrium (arrows) caused by deep myometrial invasion.
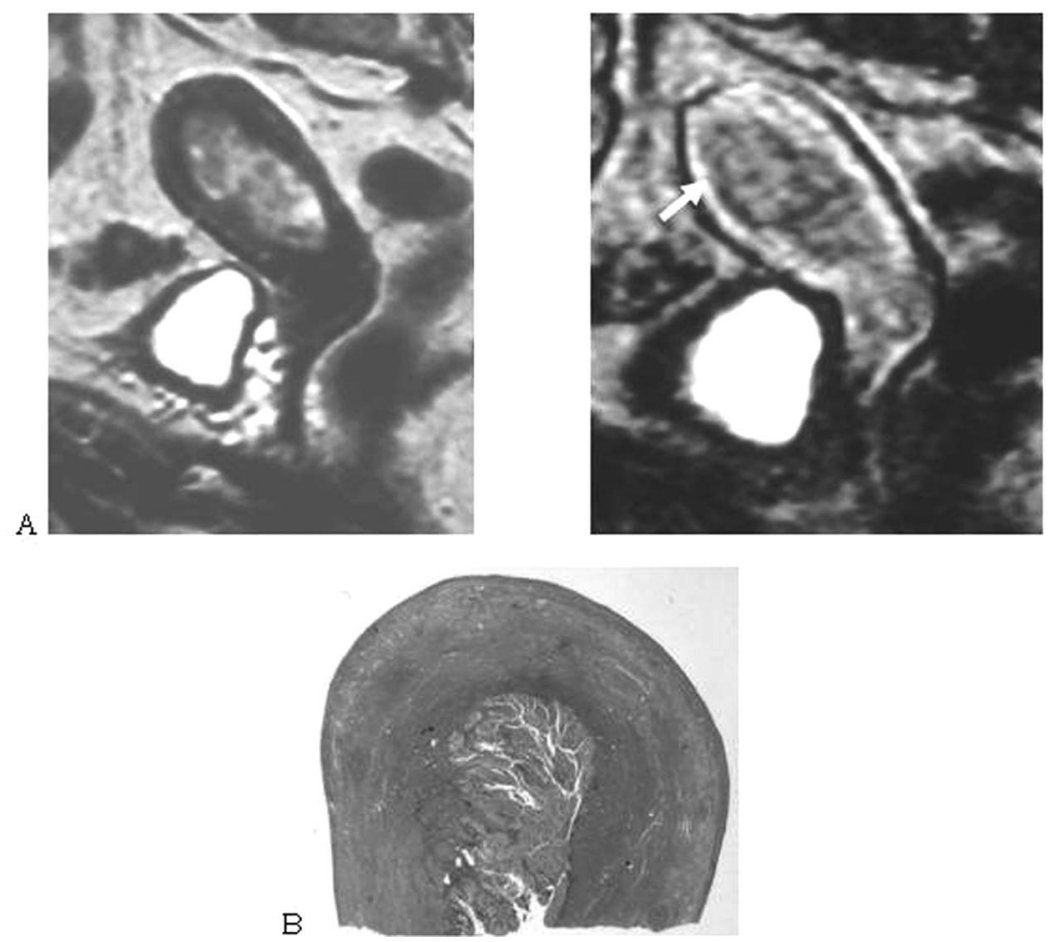

Figure 4. Endometrial carcinoma with superficial myometrial invasion in an 83-year-old woman. (A) Sagittal T2-weighted image (left) shows tumour causing segmental disruption of the junctional zone with tumour confined to the inner half of the myometrium; and sagittal dynamic enhanced image (right) shows a regular thinning of the myometrium (arrow). (B) Histological section of the uterine wall with an adenocarcinoma invading the internal half of the myometrium.

extension and/or invasion in 31 cases, including all cases identified by MRI. In the 12 patients understaged by MRI, six cases had only endocervical epithelial extension (stage IIA).
The general sensitivity, specificity, diagnostic accuracy, and positive and negative predictive values for cervical invasion was respectively: $61 \%, 100 \%, 88 \%$ and $100 \%$, $85 \%$, respectively. 

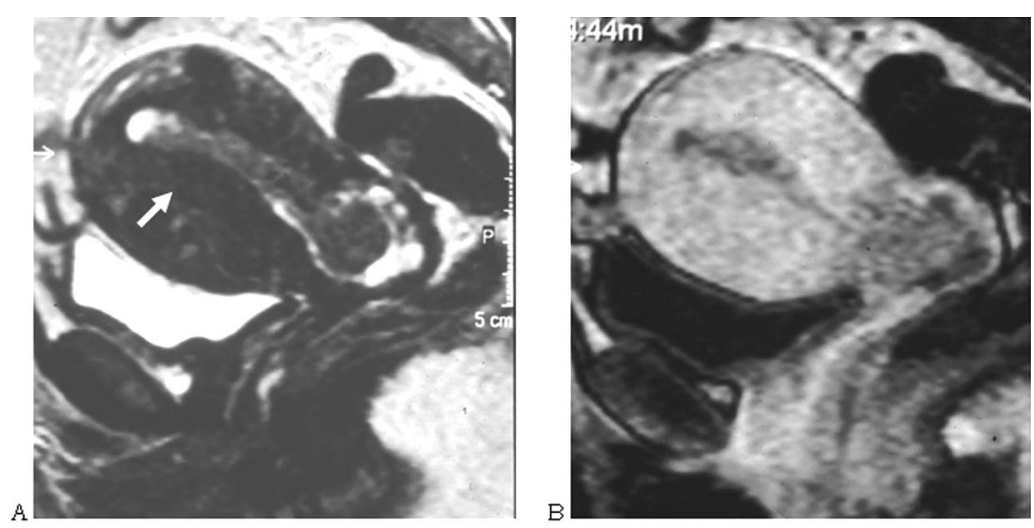

Figure 5. Images obtained in a 53-year-old woman with endocervical glandular invasion. (A) Sagittal T2-weighted image and (B) sagittal dynamic gadolinium enhanced image show the tumour in the uterine cavity with superficial myometrium invasion. Adenomyosis of the anterior wall of the uterus (arrow) is identified on T2-weighted image. We can also see the tumour extending into the cervical canal and marked widening of the internal os. A regular interface between the tumour and the cervical stroma is well documented in sagittal dynamic gadolinium enhanced image.

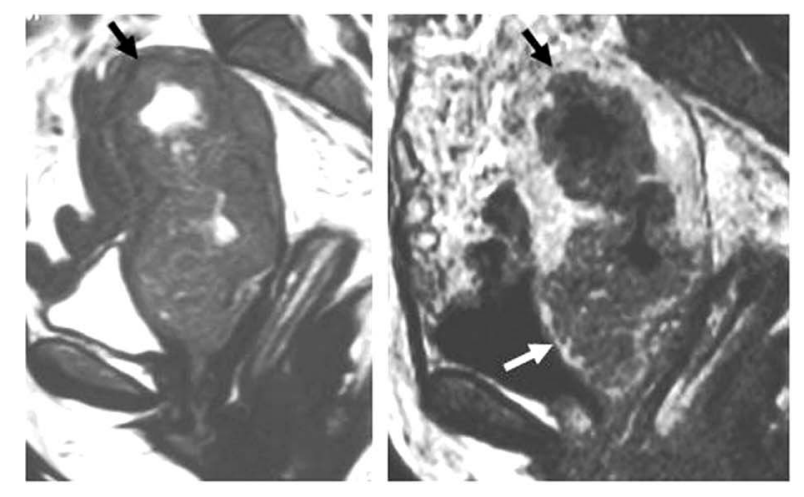

A

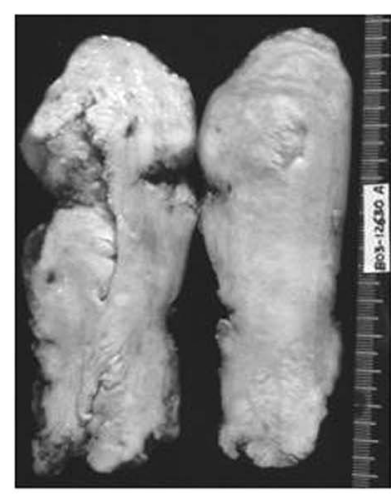

B

Figure 6. Grade 2 endometrial adenocarcinoma with deep myometrial invasion and cervical extension in a 51-year-old woman. (A) Sagittal T2-weighted image (left) and sagittal dynamic gadolinium enhanced image. The images demonstrated the tumour extending into the outer half of the myometrium (black arrows) and into the cervix. There is widening of the internal os and of the cervical canal with deep invasion of cervical stroma (white arrow). Differentiation of tumour from residual myometrium is easy to detect in the dynamic MR image. (B) Sagittal section of hysterectomy gross specimen with the tumour invading deep wall of the myometrium and with cervical stromal invasion.

\section{Discussion}

Endometrial carcinoma is the second most common female genital tract carcinoma. Treatment planning and prognosis are determined by prognostic factors that include depth of myometrial invasion, cervical extension and lymph node metastases (Frei and Kinkel 2001). A higher tumour grade correlates with lower survival rates and higher rate of myometrial invasion (Kinkel et al. 1999). The tumour grade and the depth of myometrial invasion correlate strongly with the prevalence of lymph node metastases (Frei and Kinkel 2001). As clinical staging carries an overall understaging rate of $13-22 \%$, routine surgical staging has been recommended by FIGO since 1988 (Frei and Kinkel 2001; Kinkel et al. 1999).

Surgery is the treatment of choice in patients with noninvasive or locally advanced disease (Manfredi et al. 2004). Patients with $>50 \%$ myometrial invasion have a six- to seven-fold higher prevalence of pelvic and para-aortic lymph node metastases and of advanced surgical stage (Frei et al. 2000). Therefore, patients with deep myometrial invasion have been considered for more aggressive surgical staging, which includes pelvic and para-aortic lymphadenectomy (Frei et al. 2000). Recent data from a Study in the Treatment of Endometrial Cancer (ASTEC) has shown that lymphadenectomy in addition to hysterectomy and salpingo-oophorectomy does not improve survival in early-stage endometrial carcinoma. This study was designed to assess the effect of lymphadenectomy and adjuvant external beam radiation therapy in the treatment of endometrial cancer (Kitchener 2006).

As well-established 'primary treatment in a cancer patient gives the best opportunity for cure' (Kinkel et al. 1999), accurate preoperative assessment of the depth of myometrial invasion and/or cervical invasion is very important in treatment planning (Cunha et al. 2001).

Among the imaging modalities that have been proposed for the evaluation of myometrial invasion, contrastenhanced MRI has a substantially higher sensitivity and specificity than endovaginal US and CT (Frei et al. 2000). MRI studies allow tumour visualisation, determine the degree of myometrial invasion, and also evaluate cervical extension, obtaining good accuracy in endometrial tumour evaluation.

The presence and depth of myometrial infiltration can be assessed on T2-weighted images as an interruption of the 
junctional zone, which appears with low signal intensity. However, in postmenopausal women, this could be more difficult to assess because uterine involution makes the junctional zone poorly visible and the myometrium thinner. To overcome this limitation, a dynamic study should be performed, because it can depict different enhancement times of the adenocarcinoma compared with those of the adjacent myometrium, improving the contrast resolution of the tumour and myometrium.

In this MRI study, we used both T2-weighted images and a dynamic study to evaluate the depth of myometrial invasion and cervical extension. The dynamic study was extremely helpful in those cases where the junctional zone was not clearly visualised.

Our results are in keeping with previous series (Hardesty et al. 2000; Yamashita et al. 1993; Ito et al. 1994). We obtained an accuracy of $95 \%$ in the evaluation of deep myometrial invasion and an accuracy of $88 \%$ regarding cervical extension. The high diagnostic accuracy in the assessment of deep myometrial infiltration in our series was improved because we acquired images during the equilibrium phase of the dynamic study, i.e. 90-120 s after contrast administration, according to Manfredi et al. (2004). In their study, the lesion detection was more easily achieved during the equilibrium phase, where a significantly better tumour and myometrium contrast-to-noise ratio is present than in the other phases of the dynamic study.

The presence of leiomyomas, high-grade tumour progression and the presence of small isolated tumoural foci were factors that in our opinion decreased the accuracy in our study as previously described.

In conclusion, the high accuracy achieved in this study suggests that MRI should be considered as a method for preoperative endometrial carcinoma 'radiological staging'.

\section{References}

Anonymous. 1989. FIGO stages 1988 revision. Gynecologic Oncology 35:125-127.

Belloni C, Vigano R, del Maschio A, Sironi S, Taccagni GL, Vignali M. 1990. Magnetic resonance imaging in endometrial carcinoma staging. Gynecologic Oncology 37:172-177.

Cunha TM, Félix A, Cabral I. 2001. Preoperative assessment of deep myometrial and cervical invasion in endometrial carcinoma: comparison of magnetic resonance imaging and gross visual inspection. International Journal of Gynecological Cancer 11: $130-136$
Frei KA, Kinkel K. 2001. Staging endometrial cancer: role of magnetic resonance imaging. Journal of Magnetic Resonance Imaging 13:850-855.

Frei KA, Kinkel K, Bonel HM, Lu Y, Zaloudek C, Hricak H. 2000. Prediction of deep myometrial invasion in patients with endometrial cancer: clinical utility of contrast enhanced MR imaging - a meta-analysis and Bayesian analysis. Radiology 216 : $444-449$

Glantz SA. 1981. Primer of biostatistics. New York: McGraw-Hill.

Hardesty LA, Sumkin JH, Nath ME, Edwards RP, Price FV, Chang TS et al. 2000. Use of preoperative MR imaging in the management of endometrial carcinoma: cost analysis. Radiology 215:45-49.

Hricak H, Rubinstein LV, Gherman GM, Karastaed N. 1991. MR imaging evaluation of endometrial carcinoma: results of an NCI cooperative study. Radiology 179:829-832.

Ito $K$, Matsumoto $T$, Nakada $T$, Nakamishi $T$, Fujita $N$, Yamashita H. 1994. Assessing myometrial invasion by endometrial carcinoma with dynamic MRI. Journal of Computer Assisted Tomography 18:77-86.

Kinkel K, Kaji Y, Yu KK, Segal MR, Lu Y, Powell CB et al. 1999. Radiologic staging in patients with endometrial cancer: a metaanalysis. Radiology 212:711-718.

Kitchener H. 2006. ASTEC Trial - a study in the treatment of endometrial cancer. A randomized trial of lymphadenectomy and adjuvant external beam radiotherapy. 37th Annual Meeting of the Society of Gynaecologic Oncologists of North America, Palm Springs, CA.

Landis JR, Koch GG. 1997. The measurement observer agreement for categorical data. Biometrics 33:159-174.

Lien HH, Blomlie V, Tropé C, Kaern J, Abeler VM. 1991. Cancer of endometrium: value of $\mathrm{MR}$ imaging in determining depth of invasion into the myometrium. American Journal of Roentgenology 157:1221 - 1223 .

Manfredi R, Mirk P, Maresca G, Margariti PA, Testa A, Zannoni GF et al. 2004. Local-regional staging of endometrial carcinoma: role of MR imaging in surgical planning. Radiology 231:372-378.

Sironi S, Colombo E, Villa G, Taccagni G, Belloni C, Garancini P et al. 1992a. Myometrial invasion by endometrial carcinoma: assessment with plain and gadolinium-enhanced MR imaging. Radiology 185:207-212.

Sironi S, Taccagni G, Garancini P, Belloni C, Del Maschio A. 1992b. Myometrial invasion by endometrial carcinoma: assessment by MR imaging. American Journal of Roentgenology 158: $565-569$.

Yamashita Y, Harada M, Sawada T, Takahashi M, Kohiji M, Okamura H. 1993. Normal uterus and FIGO stage I endometrial carcinoma: dynamic gadolinium-enhanced MR imaging. Radiology 186:495-501. 